\title{
Sammelrezension
}

\section{Handbücher, Wörterbücher und Lexika der Sozialen Arbeit}

Seit 2008 hat unsere jugend in lockeren Abständen zu zentralen Komplexen der Sozialen Arbeit Sammelrezensionen veröffentlicht. Zunächst als Übersichten für Studierende, aber auch für PraktikerInnnen, die für ihre Berufstätigkeit auf aktuelle Fachliteratur zurückgreifen wollen.

So zuerst zu Hand- und Wörterbüchern/Lexika (Sabine Behn/Gabriele BindelKögel in uj 11+12/2008, 502-506), dann zu Recht und Verwaltung (Dieter Kreft in uj 11+12/2010,503-506), zu Einführungen in die Soziale Arbeit (C. Wolfgang Müller in uj 5/2011, 230-237), zu Methoden (Vera Birtsch in uj 11+12/2012, 498-507) und zuletzt zur Geschichte der Sozialen Arbeit (Ralph-Christian Amthor in uj 5/2013, 220-228).

Wir hatten von vornherein die Absicht, diese Sammelbesprechungen immer dann zu wiederholen, wenn sich die Literaturlage zu diesen Komplexen erheblich verändert hat. Das ist für die Hand- und Wörterbücher/Lexika inzwischen der Fall.

\section{Über die zentralen Hand- und Wörterbücher/Lexika}

Seit 2011 sind von diesen vier Hauptwerken des Komplexes Hand-und Wörterbücher, Lexika vollständig oder mindestens grundlegend überarbeitete und aktualisierte neue Auflagen erschienen.

Hier in der Reihenfolge der Erscheinungsjahre aufgeführt:

D Dieter Kreft/Ingrid Mielenz (Hrsg.) (2013): Wörterbuch Soziale Arbeit. Aufgaben, Praxisfelder, Begriffe und Methoden der Sozialarbeit und Sozialpädagogik. 7. vollständig überarbeitete und aktualisierte Aufl. Beltz Juventa, Weinheim/Basel, $€ 68$,-

$>$ Werner Thole (Hrsg.) (2012): Grundriss Soziale Arbeit. Ein einführendes Handbuch. 4. Aufl. VS, Wiesbaden, $€ 79,95$
- Hans-Uwe Otto/Hans Thiersch (Hrsg.) (2011): Handbuch Soziale Arbeit. 4. völlig neu bearbeite Aufl. Ernst Reinhardt, München/Basel, $€ 79,90$

- Deutscher Verein für öffentliche und private Fürsorge e.V./DV (Hrsg.) (2011): Fachlexikon der sozialen Arbeit. 7. völlig überarbeitete und aktualisierte Aufl. Nomos, Baden-Baden, $€$ 44,-

Weil die Vorworte zu den Auflagen auch immer etwas über die Aktualität der Beiträge aussagen, noch diese Ergänzungen: Das Vorwort des DV ist vom November 2010 datiert, das von Otto/Thiersch vom Januar 2011, das von Kreft/ Mielenz vom Juli 2012, das von Thole ist undatiert geblieben. Die LeserInnen dieser Werke können also davon ausgehen, dass die Beiträge regelmäßig in den Jahren 2010 bis Mitte 2012 verfasst wurden. 
Inzwischen führen alle vier Werke die "Soziale Arbeit" im Titel, nur der DV schreibt das noch als „soziale Arbeit".

Von Ingrid Mielenz und Dieter Kreft wissen wir, dass sie ursprünglich (also Ende der 1970er Jahre) ihre Arbeit noch Wörterbuch Sozialarbeit/ Sozialpädagogik nennen wollten. Und nur, weil 1977 Arnold Schwendke sein Wörterbuch Sozialarbeit und Sozialpädagogik herausgab, wichen sie auf „Soziale Arbeit" aus. Otto/Thiersch nannten ihr Werk noch bis zur 3. Auflage „Handbuch Sozialarbeit/Sozialpädagogik" und sind erst mit der neuesten Auflage zu „Handbuch Soziale Arbeit" gewechselt. Diese Entwicklung deutet darauf hin, dass Soziale Arbeit inzwischen der unbestrittene Oberbegriff für Sozialarbeit/ Sozialpädagogik ist.

Anzumerken bleibt, dass der DV und Otto/ Thiersch weiterhin keinen eigenständigen Beitrag "Soziale Arbeit" vorlegen. Beim DV wird im Text ausdrücklich auf das Stichwort Sozialarbeit/ Sozialpädagogik verwiesen, bei Otto/Thiersch gibt es zwei größere Beiträge zu Theorie und Theoriegeschichte und zu Theoriekonstruktion und Positionen der Sozialen Arbeit. Kreft/Mielenz haben wie schon in den Vorauflagen in einem eigenständigen Beitrag dargestellt, was ihrer Meinung nach die Soziale Arbeit kennzeichnet, strukturiert, auszeichnet.

Werner Thole beginnt den von ihm herausgegebenen Grundriss mit einer ausführlichen Standortbestimmung zur Sozialen Arbeit (immerhin 54 Seiten), eine bemerkenswerte, fast schon eigenständige kleine Einführung in die Soziale Arbeit.

\section{Welche Positionen besetzen die einzelnen Werke?}

Lexikon: Der DV war immer sehr eindeutig, er legte und legt ein Lexikon vor, das mit über 1.500 Stichwörtern eine knappe erste Information zu allen wichtigen Begriffen der sozialen
Arbeit liefert - immer mit mindestens einem Literaturhinweis abgeschlossen, inzwischen auch mit Verweisen auf Internettexte.

Wörterbuch: Kreft/Mielenz haben auf der gedachten Horizontale der relevanten Hand- und Wörterbücher/Lexika sehr früh die nächste Position des Wörterbuches besetzt. Inzwischen 323 Beiträge (eben keine Stichwörter), gedacht als zunächst eine qualifizierte Erstinformation und dann verdichtet über bestimmte Themenblöcke auch als Einführung in die Soziale Arbeit nutzbar (so in ihrem Vorwort zu aktuellen Auflage).

Handbuch: Otto/Thiersch nannten ihre Ausgaben von Anfang an Handbücher. Sie verfolgen mit dem „Handbuch das Ziel, Soziale Arbeit in ihrer gesellschaftlichen Notwendigkeit und im Prinzip sozialer Gerechtigkeit zu fundieren ..." (Vorwort V). In den über 175 Artikeln wird der Stand der Sozialen Arbeit in ihrer theoretischen Diskussion, Forschung und Praxis beschrieben. Und sie erweitern das Handbuch fortlaufend über ein Onlineportal (www.handbuch-sozialearbeit.de) - inzwischen sind dort bereits 10 neue Artikel erschienen. Eine Entwicklung in Richtung Wörterbuch zeichnet sich ab und dadurch die Gefahr, die Ausweisung als Handbuch aufzugeben.

Grundriss als Handlbuch: Der neue Thole ist gegenüber seinen Vorauflagen um fast ein Drittel angewachsen. Hatte die 2. Auflage 2005 noch 983 Seiten, umfasst die 4. Auflage nun bereits 1.238 Seiten. Aber wie bereits in der ersten Sammelbesprechung zu diesem Komplex 2008 ausgeführt, ist der Grundriss von Thole mit seiner systematischen Ordnung ein klassisches Handbuch. Der schon erwähnten Einführung folgen in 11 Hauptkapiteln über 70 Beiträge.

\section{Vorteile/Nachteile}

Inzwischen umfassen diese vier Werke 5.261 Seiten, das alles im Detail zu besprechen, ist ausgeschlossen. Wir haben uns daher unserer 
Meinung nach zentralen Fragen zugewandt und dazu Antworten versucht. Neben der schon angesprochenen grundlegenden Konzeption (und damit möglichen Unterscheidung) sowie der ebenfalls bereits beschriebenen Aktualität sind das vor allem die Nutzerfreundlichkeit und wieder die Frage danach, ob die ganze Breite der Sozialen Arbeit angesprochen worden ist.

Nutzerfreundlichkeit: Auch der DV hat inzwischen ein Stichwortverzeichnis aufgenommen, alle anderen bieten ihren LeserInnen allerdings mehr.

Bei Otto/Thiersch finden sich gleich zu Beginn ein alphabetisches Verzeichnis der Beiträge und dazu ein sehr gelungenes systematisches Verzeichnis (also gebündelt etwa nach Geschichte, Handlungskompetenz, Methoden, Recht und Rechte). Kreft/Mielenz bieten wie in allen Vorauflagen wieder ein Stichwortverzeichnis an, das dann allerdings durch vier Anhänge komplettiert wird (Organisationen, Zeitschriften, Materialien, Soziale Arbeit im Internet) - eine außerordentlich hilfreiche Zusatzinformationen für die NutzerInnen. Thole hat die Informationsteile anders angelegt: Jedes der 11 Hauptkapitel schließt mit einem Serviceteil ab: einmal "zum Weiterlesen“ und dann mit speziellen Informationen zum Kapitelschwerpunkt wie etwa Zeitschriften, Interessenvertretungen u. a.m. Aber: Anders als in den Vorauflagen gibt es kein Gesamtstichwortverzeichnis mehr, gewiss kein Vorteil für die Nutzerlnnen.

Was darüber hinaus noch aufgefallen ist: Alle vier Titel liefern sorgfältige Autoreninformationen, aber nur bei Kreft/Mielenz ist dabei sogleich zu erkennen, welche Beiträge der/die Autorln verfasst hat. Alle liefern auch zu fast allen Stichwörtern/Beiträgen/Artikeln weiterführende Literaturhinweise, des Öfteren sind allerdings diese Literaturverweise übermäßig lang.

\section{Ist die ganze Breite der Sozialen Arbeit behandelt?}

Ja, ganz eindeutig in allen vier Titeln. Es fehlt bei keinem etwas Wichtiges, um die zentralen Begriffe Sozialstaat, Sozialpolitik, Soziale Sicherung und Soziale Arbeit (mit Alten-, Behinderten-, Gesundheits-, Kinder- und Jugendarbeit, Sozialhilfe/Grundsicherung sowie die Grundsicherung für Arbeitsuchende).

Alle Werke sind seit 1980 (DV und Kreft/Mielenz) und 1984 (damals noch als Eyfferth/Otto/ Thiersch) bzw. 2002 (Thole) am Markt und haben immer wieder neue Auflagen erfahren. Sie sind inzwischen durchstrukturiert und fachlich auf den neuesten Stand gebracht worden. Sie haben wohl auch immer wieder voneinander Anregungen erhalten und/oder voneinander profitiert.

Jedes Buch für sich vermittelt seinen Leserlnnen auf eine eigenständige Weise den Stand der Profession bzw. Disziplin Soziale Arbeit. Jeder Titel ist zugleich auch ein Beleg dafür, wie sich die Soziale Arbeit seit den 1970er Jahren, als es noch kein vergleichbares Werk gab, entwickelt hat und zu welchen fachlichen Standards für die Sozialarbeit/Sozialpädagogik diese Entwicklungen geführt haben.

Jedenfalls sehen wir jedes Buch für sich als einen beachtlichen Beweis für diese These.

\section{Was bleibt als Empfehlungen?}

Für Studierende, die zu Beginn viele Fragen haben, die noch suchen, unsicher sind, was sie wirklich interessiert, denen empfehlen wir das Fachlexikon der sozialen Arbeit des Deutschen Vereins, weil sie darin erst einmal kurz und knapp alles rund um die Soziale Arbeit finden.

Wer dann schon etwas genauer fokussieren kann und in der Lage ist, seine Interessenschwerpunkte präziser zu bestimmen, wer vielleicht im Bachelor-Studium immer wieder Prüfungen vorzubereiten hat, den kann gewiss das 
Wörterbuch von Kreft/Mielenz bei seinen Arbeiten sehr gut unterstützen.

Wer Vertiefungen zu bestimmten Themen sucht, der kann auf den Grundriss Soziale Arbeit von Thole zurückgreifen. Wer schließlich eine zunehmend theoretische Fundierung für seine Studieninteressen erreichen möchte, der wird dabei regelmäßig durch das Handbuch von Otto/Thiersch unterstützt werden.

Also: Alle vier Titel haben ihren eigenständigen Wert, sind auf der Höhe der Fachdiskussion und weitgehend aktuell (so weit das bei der Schnelllebigkeit der sozialen Veränderungen überhaupt möglich ist). Allerdings werden (sollten) wohl Praktikerlnnnen, die sich neben ihrem täglichen Tun für neue Entwicklungen und andere Arbeitsfelder interessieren, eher zu den beiden Titeln des DV und von Kreft/Mielenz greifen und darin nach Antworten auf ihre Fragen suchen, denn sie sind von ihrer Anlage und Diktion her regelmäßig den besonderen Bedürfnissen der Praxis etwas näher als die anderen Werke.

\section{Ein kleiner Nachtrag}

WernerThole, Davina Höblich und Sarina Ahmed haben 2012 bei Klinkhardt UTB ein Taschenwör- terbuch Soziale Arbeit herausgegeben (€ 17,99). Die Herausgeberlnnen wollten die vorliegenden sozialpädagogischen Nachschlagewerke um ein handliches, alltagskompatibles Kompendium erweitern. Auf 320 UTB- Seiten ist ein gelungenes Nischenprodukt entstanden, mit immerhin 330 Stichwörtern über Fachbegriffe, Ziele, Aufgaben und Fragestellungen der Sozialen Arbeit.

Die Arbeit ist offenbar ein Nebenprodukt des dreibändigen Klinkhardt-Lexikons Erziehungswissenschaft, aber leider ist uns beim Blättern aufgefallen, dass offenbar zu vielen Beiträgen die Literaturhinweise nicht fortgeschrieben und aktualisiert wurden. Aber anders als die anderen besprochenen Titel passt dieser wohl in eine Tasche, während das bei den anderen zuvor besprochenen immer schwerer wird: Wir haben auf einer Briefwaage festgestellt, dass der Thole inzwischen 2,2 kg, Kreft/Mielenz 1,8 kg, Otto/ Thiersch immerhin noch 1,7 kg und selbst das dagegen fast bescheiden auftretende Fachlexikon des DV noch $926 \mathrm{~g}$ auf die Waage bringt.

Sabine Behn und Gabriele Bindel-Kögel 